\title{
The Sars-Cov-2 Pandemic and the Brave New Digital World of Environmental Enrichment to Prevent Brain Aging and Cognitive Decline
}

\author{
H. Hampel ${ }^{1}$, A. Vergallo ${ }^{1}$ \\ Eisai Inc., Neurology Business Group, Woodcliff Lake, NJ, USA
}

Corresponding Author: Harald Hampel and Andrea Vergallo, Eisai Inc., Neurology Business Group, 100 Tice Blvd, Woodcliff Lake, NJ 07677, USA, Tel: (+1) 201-746-2060 (o) harald_hampel@eisai.com; andrea_vergallo@eisai.com

\begin{abstract}
Individuals experiencing brain aging, cognitive decline, and dementia are currently confronted with several more complex challenges due to the current Sars-Cov-2 pandemic as compared to younger and cognitively healthy people. During the first six months of the pandemic, we are experiencing critical issues related to the management of mild cognitive impairment (MCI) and dementia. The evolving, highly contagious global viral spread has created a pressure test of unprecedented proportions for the existing brain health care infrastructure and related services for management, diagnosis, treatment, and prevention. Social distancing and lock-down measures are catalyzing and accelerating a technological paradigm shift, away from a traditional model of brain healthcare focused on late symptomatic disease stages and towards optimized preventive strategies to slow brain aging and increase resilience at preclinical asymptomatic stages. Digital technologies transform global healthcare for accessible equality of opportunities in order to generate better outcomes for brain aging aligned with the paradigm of preventive medicine.
\end{abstract}

Key words: Brain aging, Alzheimer's disease, enviromental enrichment, social distancing.

\section{Introduction}

S tudies using experimental models of Alzheimer's disease (AD) indicate that multi-sensory cognitive stimulation, framed as "environmental enrichment", is associated with transient improvement and a long-lasting slowed decline of spatial memory as well as reduced accumulation of cerebral amyloid $(1,2)$. Some studies further suggest that an enriched environment can induce (epi)genetic changes that support hippocampal neurogenesis and synaptic plasticity (3). Overall, cognitive stimulation shows promising potential as a viable neuroprotective strategy for the early preclinical asymptomatic stages of AD (4).

Morever, environmental enrichment has the potential to increase brain resilience to stress and support compensatory capacity to incipient pathophysiological mechanisms in a time-related fashion (5-7). It is well Received June 10, 2020 established that human face-to-face interaction and different patterns of social stimuli significantly contribute to cognitive reserve (8), in healthy aging individuals as well as in patients with mild cognitive impairment and dementia $(8,9)$.

On the other hand, large-scale human studies showed that an environment that is deprived of stable cognitive stimulations, such as social interaction, represents a risk factor for cognitive impairment and decline in patients suffering from dementia or individuals with MCI and even cognitively "healthy" people $(5,7,8)$. For instance, people suffering from dementia and who are institutionalized in nursing homes, not only have a high risk of infectious diseases and general medical health deterioration but are at increased risk of cognitive- worsening as well. This is due to a reduction of psychosocial and physical activities as a consequence of prolonged social isolation, spatial confinement and physical disconnection from society, family and friends. In an at-home setting, cognitively healthy older adults with age-related, lifestyle and medical risk factors of cognitive decline or individuals with incipient MCI may have faster and, in some cases steeper, cognitive and psycho-behavioral decline. Such a dynamic is also a consequence of the loss of social connections, personal engagement and environmental stimuli that support resilience to pathophysiological factors inducing brain network dysfunctions.

Prolonged psychosocial distancing and physical-spatial confinement create fertile soil for an emerging mental health crisis extending to a large number of people that may experience chronic stress, depression, anxiety, and feeling of isolation, with insufficient access to mental health care (10). Such an emotional-affective impact of the pandemic is detrimental for the vulnerable population in general by sharpening pre-existing psychic symptoms, including substance abuse, but also for individuals with cognitive decline or dementia.

In addition to cognitive deficits, patients with $\mathrm{AD}$ may experience a variety of neuropsychiatric symptoms, behavioral and psychological symptoms (BPSD), which include emotional, perceptive, and behavioral 
domains (11-13). The occurrence of BPSD such as apathy, depression, anxiety, and sleep-wake disorders may precede overt cognitive decline and negatively influence disease prognosis, consequently aggravating the socialmedical-economic burden of dementia in regional and global healthcare services, society at large and in communities and families (11-13).

In summary, Covid-19 pandemic and related restrictive measures - including social distancing and shelter-inplace programs - create extreme life conditions with prolonged periods of severe social inactivity, deprivation, and cognitive-behavioral, as well as psychosocial understimulation that may, trigger, and drive significant behavioral and psychosocial maladaptations coupled with the decline of cognitive performance in people at risk of $\mathrm{AD}$ or already in early or advanced stages of the disease $(14,15)$. Therefore, the pandemic represents an acute significant psycho-social-medical brain system challenge for all individuals affected by or at risk of AD (and other neurodegenerative dementias) across all stages of the disease to continuum, from the management of healthy brain aging preclinical $\mathrm{AD}, \mathrm{MCI}$, and dementia $(14,15)$.

\section{The current challenges}

All existing prediction models for the next 30 years indicate an exponential increase of dementia sufferers associated with dramatically rising direct and indirect costs. These studies have not considered the increased social-medical-economic costs that any large-scale global catastrophe, such as the current pandemic, may significantly add to the numbers of patients and costs. Reliable models and projections regarding the development of the SARS-Cov-2 viral transmission, show that the pandemic i) will be a long-lasting process with expected multiple outbreaks (with regional and global waves, peaks, valleys, slow, medium or fast burn) requiring mitigation measures, such as social distancing and lock-down for the next year at least and ii) will likely require very flexible adaptive changes in how the general public may engage in healthcare pathways (16). It is arguable that the pandemic and the related barriers to social interaction and environmental stimulation may be detrimental to brain aging and accelerate the behavioral-cognitive decline, progression, and conversion to a dementia syndrome of more $\mathrm{MCI}$ individuals than expected by previously reported results. Regardless of the duration of the pandemic, the current situation provides a sufficient basis for practical reflection and analysis in order to identify several shortcomings and pitfalls in the prevention and management model of agerelated cognitive decline. In the short-term, the currently established structure of many regional healthcare systems across the country will be overloaded. The onslaught of the first pandemic wave has exposed the vulnerability of the existing healthcare infrastructure, which has been challenged to accomodate the increased patient demand and, in particular, with patients suffering from advanced cognitive impairment and dementia. All stakeholders need to develop integrated comprehensive and strategic adaption and mitigation plans to anticipate a potentially aggravated situation of a combined cognitive pandemic on top of the viral pandemic, potentially even worse during winter times with additional convergence with flu epidemic transmissions.

\section{Future perspectives and considerations}

A viable and swift solution to cope with the risk of decompensation and loss of control regarding the global healthcare management of brain aging, MCI and dementia may come through the leveraging of traditional and emerging breakthrough smart technologies, including consumer electronics, internet-based distant communication, and remote virtual reality $(15,17,18)$. The implementation of these technological innovations is clearly on the horizon and will eventually enrich and optimize the AD patient journey and the diagnostic pathway. For instance, globally accessible quantitative and scalable measures (predictive analytics), of subtle lifestyle changes can provide predictive biomarkers of reduced cognitive and psycho-behavioral performance, even in community-based and at-home settings $(17,19)$.

A promising technology based wearable analytics such as actigraphy is advancing to clinical validation to monitor daily life motor activity in aging and cognitively impaired individuals and to gather multi-dimensional information, sleep activity, apathetic syndrome signs, etc (20-22). In addition, a broad set of smartphones applications are under development to monitor daily-life physical and cognitive performance and impacting the longitudinal evolution using interactive entertainment and game-oriented platforms $(23,24)$. The idea of digital biomarkers for short-term and long-term digital phenotyping of cognitive-behavioral performance will enter clinical practice in the near future.

The current pandemic, has created the need for healthcare providers need to develop "therapeutic" tools to foster and preserve social interactions between older adults, patients, and caregivers that stabilize and maintain the individual's quality of life and independence as long as possible and delay the onset and progression of cognitive decline and increasing dependency with ultimate institutionalization (regression to nursing home placement).

Small and large-scale companies across different industries sectors have adopted smart and innovative home-working solutions by leveraging digital communication channels and replacing live conferences, meetings, and conventional on-site work. Employees worldwide have experienced micro-environmental shifts to accommodate the demand for increased flexibility catalyzed by the pandemic. The question arises naturally; 
can these same digital communication channels be utilized and employed for older adults to optimize visual and auditory stimulation while incorporating digital socialization for digital environmental enrichment and preservation of cognitive reserve? This paradigm would not only circumvent some or all of the pandemic-related constraints but could also represent a new generation of interventions to effectively counteract the dynamics of brain aging.

Based on emerging evidence, we argue that a specific set of digital communication channels may be used to build up targeted collective psychosocial experiences to adopt in the community, in at-home, assisted living, or in nursing home settings with relevant benefits on cognition, mood and behavior $(25,26)$. Digital communication channels may be used to shift to, in a digital social context those traditional (i.e., in-person) cognitive stimulation programs that are associated with better cognitive reserve outcomes (27). Moreover, computer- and internet-based social interaction strategies can be used to keep older adults connected to remote loved ones and may include multi-generational participants, thus empowering and prolonging their proactive engagement in society (28). There is currently an attractive opportunity of integrating not only business but also social relationships with a more digital domain. Digital human sociality has been proposed as a tool to counteract feelings of loneliness, a risk factor for anxiety disorders, substance abuse disorders, depression, Alzheimer's disease, and cognitive decline in general $(29,30)$. The potential gain in environmental enrichment and "downstream" cognitive and psycho-behavioral benefit through digital social networking is of significant magnitude.

For the long-term perspective, digital interactive communication platforms, coupled with emerging digital cognitive tools, may offer a multi-dimensional «omnichannel» framework for individualized stimulation and training programs tailored to the individual person's cognitive abilities and medical features. The program could be managed remotely by upgrading applications in any type of hardware, i.e., smartphones, smart TVs, or tablets, including tests to improve visual-spatial abilities, executive and memory functions, among others. Digital music and art therapy programs could be used for selective multi-domain training, including language (31). In parallel, music therapy could also be used in the community and in nursing homes, for its positive effects on BPSD (32).

Computer-driven simulations replicating reallife environments, i.e., virtual reality, are being tested to create a virtually enriched environment with individualized multisensory experiences that may substantially foster cognitive reserve (33-35). Another dimension of interactive experiences for new-generation and social distancing-compatible therapeutic strategies related to dementia comes from computer game-based interventions which have shown potential to induce psycho-physical benefits, including improvements on multi-domain cognitive abilities $(23,36)$. Poor physical activity is an established risk factor for age-related cognitive decline (see Figure 1) (5.) Therefore, digital social stimuli coupled with customized physical activity, for instance personal programs with treadmills / bikes connected with social networks, may represent a viable synergistic strategy to enrich at-home or nursing homes settings to preserve cognitive reserve in older adults while they age.

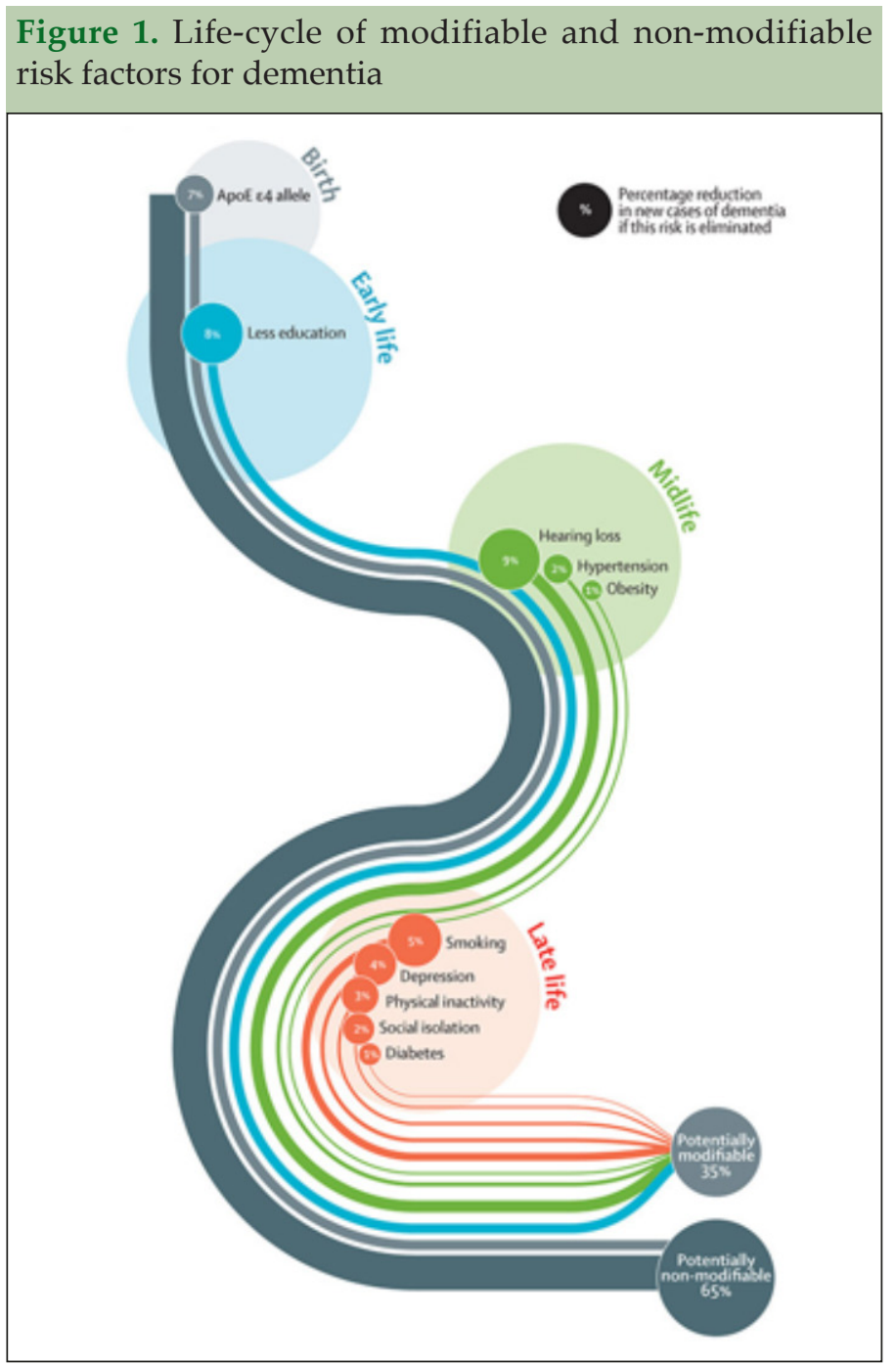

Edited from "Dementia prevention, intervention, and care. The Lancet, July 20, 2017 [https: / / www.thelancet.com/commissions/dementia2017]

\section{Conclusions}

We think that all available digital technologies can provide smart solutions to counteract the aftermath of the pandemic, i.e., reduction of psychosocial and physical activities because of prolonged social isolation, spatial confinement and physical disconnection from other people, including family, friends, coworkers, etc.

Some challenges must be addressed before digital 
environmental enrichment programs are set in motion. These include addressing ethical issues related to the collection of data, instituting a public policy that benefits the broader community, accessibility to the internet, financing digital platforms and networks. Moreover, older adults, individuals with MCI, and patients with dementia would need to be systematically trained to adequately use the available tools for social interaction and cognitive stimulation.

We promote tight collaboration among academia, pharma industry, healthcare, advocacy, policy, and regulatory stakeholders to facilitate the implementation of a digital environmental enrichment into clinical practice for new-generation preventive strategies of dementia

The right time for a holistic model of medicine, that encompasses the whole patient's psycho-physical and social network, to prolong healthspan and not only to treat symptoms and diseases, has came. In this context, digital technologies can create fertile soil for globally accessible equal opportunities for improving brain aging outcomes aligned with the paradigm of preventive medicine.

Conflict of interest: $\mathrm{HH}$ is an employee of Eisai Inc. and serves as Senior Associate Editor for the Journal Alzheimer's \& Dementia and does not receive any fees or honoraria since May 2019; before May 2019 he had received lecture fees from Servier, Biogen and Roche, research grants from Pfizer, Avid, and MSD Avenir (paid to the institution), travel funding from Eisai, Functional Neuromodulation, Axovant, Eli Lilly and company, Takeda and Zinfandel, GE-Healthcare and Oryzon Genomics, consultancy fees from Qynapse, Jung Diagnostics, Cytox Ltd., Axovant, Anavex, Takeda and Zinfandel, GE Healthcare, Oryzon Genomics, and Functional Neuromodulation, and participated in scientific advisory boards of Functional Neuromodulation, Axovant, Eisai, Eli Lilly and company, Cytox Ltd., GE Healthcare, Takeda and Zinfandel, Oryzon Genomics and Roche Diagnostics. He is co-inventor in the following patents as a scientific expert and has received no royalties: - In Vitro Multiparameter Determination Method for The Diagnosis and Early Diagnosis of Neurodegenerative Disorders Patent Number: 8916388; • In Vitro Procedure for Diagnosis and Early Diagnosis of Neurodegenerative Diseases Patent Number: 8298784; • Neurodegenerative Markers for Psychiatric Conditions Publication Number: 20120196300; • In Vitro Multiparameter Determination Method for The Diagnosis and Early Diagnosis of Neurodegenerative Disorders Publication Number: 20100062463; • In Vitro Method for The Diagnosis and Early Diagnosis of Neurodegenerative Disorders Publication Number: 20100035286; • In Vitro Procedure for Diagnosis and Early Diagnosis of Neurodegenerative Diseases Publication Number: 20090263822 , - In Vitro Method for The Diagnosis of Neurodegenerative Diseases Patent Number: 7547553; • CSF Diagnostic in Vitro Method for Diagnosis of Dementias and Neuroinflammatory Diseases Publication Number: 20080206797; • In Vitro Method for The Diagnosis of Neurodegenerative Diseases Publication Number: 20080199966; • Neurodegenerative Markers for Psychiatric Conditions Publication Number: 20080131921; AV is an employee of Eisai Inc. He does not receive any fees or honoraria since November 2019. Before November 2019, he had received lecture honoraria from Roche, MagQu LLC, and Servier.

Acknowledgment: We thank Ricky Kurzman and Stephanie Cropper for their kind support in the critical revision of the article.

\section{References}

1. Beauquis J, Pavía P, Pomilio C, et al. Environmental enrichment prevents astroglial pathological changes in the hippocampus of APP transgenic mice, model of Alzheimer's disease. Exp Neurol. United States; 2013;239:28-37.

2. Hu Y-S, Xu P, Pigino G, Brady ST, Larson J, Lazarov O. Complex environment experience rescues impaired neurogenesis, enhances synaptic plasticity, and attenuates neuropathology in familial Alzheimer's disease-linked APPswe/ PS1DeltaE9 mice. FASEB J Off Publ Fed Am Soc Exp Biol. 2010;24:1667-1681.

3. Barak B, Shvarts-Serebro I, Modai S, et al. Opposing actions of environmental enrichment and Alzheimer's disease on the expression of hippocampal microRNAs in mouse models. Transl Psychiatry. 2013;3:e304.
4. Jankowsky JL, Melnikova T, Fadale DJ, et al. Environmental enrichment mitigates cognitive deficits in a mouse model of Alzheimer's disease. J Neurosci. 2005;25:5217-5224.

5. Livingston G, Sommerlad A, Orgeta V, et al. Dementia prevention, intervention, and care. Lancet (London, England). England; 2017;390:26732734 .

6. McEwen BS. Physiology and neurobiology of stress and adaptation: central role of the brain. Physiol Rev. United States; 2007;87:873-904.

7. Barulli D, Stern Y. Efficiency, capacity, compensation, maintenance, plasticity: emerging concepts in cognitive reserve. Trends Cogn Sci. 2013;17:502-509.

8. Stern Y. Cognitive reserve in ageing and Alzheimer's disease. Lancet Neurol 2012;11:1006-1012.

9. Cohen-Mansfield J, Thein K, Dakheel-Ali M, Regier NG, Marx MS. The value of social attributes of stimuli for promoting engagement in persons with dementia. J Nerv Ment Dis. 2010;198:586-592.

10. Holmes EA, O'Connor RC, Perry VH, et al. Multidisciplinary research priorities for the COVID-19 pandemic: a call for action for mental health science. The lancet Psychiatry. 2020;7:547-560.

11. Cerejeira J, Lagarto L, Mukaetova-Ladinska EB. Behavioral and psychological symptoms of dementia. Front Neurol. 2012;3:73.

12. Lyketsos CG, Carrillo MC, Ryan JM, et al. Neuropsychiatric symptoms in Alzheimer's disease. Alzheimers. Dement. 2011. p. 532-539.

13. Lyketsos CG, Lopez O, Jones B, Fitzpatrick AL, Breitner J, DeKosky S. Prevalence of neuropsychiatric symptoms in dementia and mild cognitive impairment: results from the cardiovascular health study. JAMA. United States; 2002;288:1475-1483.

14. Lima CKT, Carvalho PM de M, Lima I de AAS, et al. The emotional impact of Coronavirus 2019-nCoV (new Coronavirus disease). Psychiatry Res. 2020. p. 112915.

15. Ousset PJ, Vellas B. Viewpoint: Impact of the Covid-19 Outbreak on the Clinical and Research Activities of Memory Clinics: An Alzheimer's Disease Center Facing the Covid-19 Crisis. J Prev Alzheimer's Dis. 2020;7:197-198.

16. Kissler SM, Tedijanto C, Goldstein E, Grad YH, Lipsitch M. Projecting the transmission dynamics of SARS-CoV-2 through the postpandemic period. Science. 2020;368:860-868.

17. Gold M, Amatniek J, Carrillo MC, et al. Digital technologies as biomarkers, clinical outcomes assessment, and recruitment tools in Alzheimer's disease clinical trials. Alzheimer's Dement (New York, N Y). 2018;4:234-242.

18. Pinzón-Pulido S, Padial-Espinosa M, López-Samaniego L, et al. Creation Process of the Digital Platform to Foster Healthy and Active Aging: enbuenaedad. Front public Heal. 2019;7:22.

19. Kourtis LC, Regele OB, Wright JM, Jones GB. Digital biomarkers for Alzheimer's disease: the mobile/ wearable devices opportunity. NPJ Digit Med. 2019;2.

20. Ju Y-ES, Holtzman DM. Sleep evaluation by actigraphy for patients with Alzheimer disease--reply. JAMA Neurol. United States; 2013. p. 1074-1075.

21. Mulin E, Zeitzer JM, Friedman L, et al. Relationship between apathy and sleep disturbance in mild and moderate Alzheimer's disease: an actigraphic study. J Alzheimers Dis. Netherlands; 2011;25:85-91.

22. David R, Mulin E, Friedman L, et al. Decreased daytime motor activity associated with apathy in Alzheimer disease: an actigraphic study. Am J Geriatr psychiatry Off J Am Assoc Geriatr Psychiatry. England; 2012;20:806814.

23. Anguera JA, Boccanfuso J, Rintoul JL, et al. Video game training enhances cognitive control in older adults. Nature. 2013;501:97-101.

24. Tarnanas I, Tsolaki A, Wiederhold M, Wiederhold B, Tsolaki M. Fiveyear biomarker progression variability for Alzheimer's disease dementia prediction: Can a complex instrumental activities of daily living marker fill in the gaps? Alzheimer's Dement (Amsterdam, Netherlands). 2015;1:521-532.

25. Padala SP, Jendro AM, Orr LC. Facetime to reduce behavioral problems in a nursing home resident with Alzheimer's dementia during COVID-19. Psychiatry Res. 2020. p. 113028

26. Chopik WJ. The Benefits of Social Technology Use Among Older Adults Are Mediated by Reduced Loneliness. Cyberpsychol Behav Soc Netw. 2016;19:551-556.

27. Orrell M, Yates L, Leung P, et al. The impact of individual Cognitive Stimulation Therapy (iCST) on cognition, quality of life, caregiver health, and family relationships in dementia: A randomised controlled trial. PLoS Med. 2017;14:e1002269.

28. Nowland R, Necka EA, Cacioppo JT. Loneliness and Social Internet Use: Pathways to Reconnection in a Digital World? Perspect Psychol Sci a J Assoc Psychol Sci. United States; 2018;13:70-87.

29. Donovan NJ, Wu Q, Rentz DM, Sperling RA, Marshall GA, Glymour MM. Loneliness, depression and cognitive function in older U.S. adults. Int J Geriatr Psychiatry. 2017;32:564-573.

30. Wilson RS, Krueger KR, Arnold SE, et al. Loneliness and risk of Alzheimer disease. Arch Gen Psychiatry. United States; 2007;64:234-240.

31. Tomaino CM. Effective music therapy techniques in the treatment of nonfluent aphasia. Ann N Y Acad Sci. United States; 2012;1252:312-317.

32. Clements-Cortes A, Bartel L. Are We Doing More Than We Know? Possible Mechanisms of Response to Music Therapy. Front Med. 2018;5:255.

33. Bellassen V, Iglói K, de Souza LC, Dubois B, Rondi-Reig L. Temporal 
order memory assessed during spatiotemporal navigation as a behavioral cognitive marker for differential Alzheimer's disease diagnosis. J Neurosci. 2012;32:1942-1952.

34. Jebara N, Orriols E, Zaoui M, Berthoz A, Piolino P. Effects of enactment in episodic memory: a pilot virtual reality study with young and elderly adults. Front Aging Neurosci. 2014;6:338.
35. Sabbagh MN, Boada M, Borson S, et al. Early Detection of Mild Cognitive Impairment (MCI) in an At-Home Setting. J Prev Alzheimer's Dis. Switzerland; 2020;7:171-178.

36. Nouchi R, Taki $Y$, Takeuchi $H$, et al. Brain training game improves executive functions and processing speed in the elderly: a randomized controlled trial. PLoS One. 2012;7:e29676. 